\title{
Colicin Diversity: a Result of Eco-evolutionary Dynamics
}

\author{
Ludo Pagie* and Paulien Hogeweg \\ Department of Bioinformatics, Utrecht University, Padualaan 8, 3584 CH Utrecht, \\ The Netherlands
}

(Received on 15 May 1998, Accepted in revised form on 22 September 1998)

Colicins are plasmids that are carried in Escherichia coli. They code for a toxic protein and for proteins that confer on the host immunity against this toxin. When bacteria carry plasmids their growth rate is reduced. At the same time, the production of toxins makes it possible for colicinogenic bacteria to invade bacterium strains that are not immune. In natural bacterium populations there is a high diversity of colicin types. The reason for the maintenance of this diversity has been the subject of much recent debate.

We have studied a simple eco-evolutionary model of the interaction of bacteria with colicins and show that high diversity of colicins is to be expected. We find two different dynamical modes each with a high diversity: a hyperimmunity mode and a multitoxicity mode. Bacteria are immune to most toxins in the first mode but in fact produce very few toxins. In the second mode bacteria are immune only to those toxins that they actually produce. In the second mode toxin levels per bacterium are much higher, whereas immunity levels per bacterium are lower.

(C) 1999 Academic Press

\section{Introduction}

Colicin plasmids are carried in Escherichia coli and related bacteria (Pugsley, 1984; Konisky, 1982; Luria \& Suit, 1987; Hardy, 1975). The plasmids code for a toxin that kills bacteria. The plasmids also code for proteins that neutralise the toxic protein, thereby conferring immunity on bacteria that carry the plasmid. Many types of colicin plasmids exist; they are generally cross-sensitive [but see Cooper \& James (1984), Lau et al. (1984) for exceptions].

Natural E. coli populations are often found to carry colicin plasmids (Hartl \& Dykhuizen, 1984; Riley \& Gordon, 1992; Achtman et al., 1983;

*Author to whom all correspondence should be addressed.

E-mail: pagie@encode.bio.uu.nl
Singh et al. 1989). The degree of polymorphism of colicins found in natural bacterium populations is much higher than one might expect (James et al., 1996; Riley, 1993; Dykes \& Hastings, 1997). On the one hand, plasmids reduce the lowered maximum growth rates of their host. As a result plasmid-carrying bacteria should be outcompeted by wild-type bacteria. On the other hand, colicinogenic bacterium strains produce toxins that kill closely related bacteria. This gives colicin producing bacterium strains an advantage in that they produce "anti-competitor" molecules that kill bacteria competing in the same environment for the same resource. The question remains why the observed polymorphism of colicin plasmids is so high. Many attempts to answer the question are based on the additional fitness-enhancing properties 
that are supposedly carried by the plasmids (Bouma \& Lenski, 1988; Dykes \& Hastings, 1997; Feldgarden et al., 1995). Coexistence of several types of colicinogenic bacteria would in this case be possible in habitats with different ecological niches (Frank, 1994).

Previous studies on the competition between bacteria that carry different plasmids have shown that the outcome of competition in an aqueous culture, i.e. a well-mixed environment, depends on the initial frequency of the competing strains (Chao \& Levin, 1981; Levin, 1988). Competition, however, always yields one winner. In spatially explicit environments, on the other hand, colicinogenic bacteria have an overall competitive advantage over wild-type bacteria, even if the colicinogenic bacteria are initially rare (Chao \& Levin, 1981; Iwasa et al., 1998). Of course, the natural habitat of $E$. coli (the lower intestine of warm-blooded animals) is certainly a spatially structured environment (Hartl \& Dykhuizen, 1984).

Durrett \& Levin (1997) have shown that in a spatially explicit but otherwise single niche model it is possible for wild-type bacteria and colicinogenic bacteria to coexist. The condition for coexistence is that different types of bacteria exist such that each bacterium strain is capable of outcompeting another strain. In the model developed by Durrett \& Levin two colicinogenic bacteria coexist with one wild-type bacterium. The two plasmid types are cross-immune but one plasmid type produces less or no toxin and thereby imposes a lower growth penalty on its host bacterium. The latter, cheating plasmid outcompetes the more toxic plasmid but is in turn outcompeted by the wild-type bacterium. Although this transitive cycle of bacterium states does not result in the stable coexistence of the three bacterium types in a well-mixed environment, stable coexistence is easily achieved in a spatial explicit environment.

Experimental data suggest that many bacteria are immune to colicins that they do not produce (Riley \& Gordon, 1992). This immunity without toxin production may be the result of carrying plasmids that code for a second immunity protein but do not code for the corresponding toxin (Cooper \& James, 1984; Lau et al., 1984), or bacteria can become insensitive to colicins through an alteration in the membrane receptors (Luria \& Suit, 1987; Hardy, 1975). Bacteria that are immune to toxins they do not produce themselves can play a role that resembles that of the "cheating" plasmids in the model of Durrett \& Levin.

We study a spatially explicit multi-plasmid eco-evolutionary model of bacteria and colicin plasmids in order to investigate further whether space is a sufficient condition for the maintenance of a high diversity of colicin plasmids and how the interaction between plasmids and bacteria affects their ecological dynamics. We will show that the dynamics are such that a high diversity of plasmids is easily maintained in a single niche.

\section{Model}

The model is based on the interactions between bacteria and colicin plasmids, implemented in a cellular automaton with asynchronous updating. The model-bacteria are very simple entities that grow and die, depending on their own growth dynamics and on the presence of plasmids within the bacteria and in the eight neighbouring bacteria. The model-plasmids grow and die along with their host bacterium. In addition they can be generated and deleted by means of mutations.

The model contains nine different types of plasmids, i.e. nine different toxin-immunity pairs. We allow no cross-immunity between plasmid types. If a bacterium carries a plasmid type we do not specify how many plasmids of that type are present per bacterium. Plasmids are generated through mutation with a rate $\mu_{g}$ and are deleted with a rate $\mu_{d}$ (see Table 1).

A single plasmid specifies two genes: a toxin gene and an immunity gene. The two genes can mutate independently, i.e. the state of a gene can be active or inactive. Thus, plasmids can be in four states, only two of which will be considered. In a plasmid both toxin and immunity genes can be active or a plasmid can have an active immunity gene only. In the first case the host bacterium produces the toxic protein and the proteins that confer immunity. In the latter case the bacterium produces only the immunity protein. The case in which only the toxin gene is active is not taken into consideration since it 
TABLE 1

Default values for the parameters used in the simulations. $\mathrm{G}_{\mathrm{t}}$ is the number of toxin genes in a bacterium, $\mathrm{G}_{\mathrm{i}}$ is the number of immunity genes per bacterium. See text for explanations of the parameters

\begin{tabular}{lc}
\hline Parameter & Value \\
\hline Grid-size & $300 \times 300$ \\
Bacterium death rate & 0.1 \\
Add. death per toxin & 0.3 \\
Immunity growth penalty $\left(P_{i}\right)$ & $0.02-0.20$ \\
Toxin growth penalty $\left(P_{t}\right)$ & $0.02-0.07$ \\
Bacterium growth rate & $1-G_{t} \times P_{t}-G_{i} \times P_{i}$ \\
Degenerative mutation rate $\left(\mu_{d}\right)$ & $10^{-4}$ \\
Generative mutation rate $\left(\mu_{g}\right)$ & $10^{-7}$ \\
Colicin types & $\mathrm{g}$ \\
\hline
\end{tabular}

is not a viable situation; the bacterium host would be killed by the plasmid instantaneously. Since the case in which both genes are inactive is equivalent to the case in which the plasmid itself is absent the cases will be treated identically. Thus, a bacterium can carry between 0 and nine toxin genes and between 0 and nine immunity genes. Of course, a viable bacterium will carry at least as many immunity genes as it carries toxin genes and possibly more.

Although in the remainder of the paper we will generally talk about (plasmids carried by) single bacteria the model could just as well be interpreted at the level of monomorphic bacterium strains. In fact, natural bacterium hosts that secrete toxin die in the process as a result of cell lysis. Thus, being toxic to conspecifics could only be attained by "suicide". However, in a natural, monomorphic strain of colicinogenic bacteria only a small proportion of the bacteria produce toxin and are thereby killed (Pugsley, 1984; Luria \& Suit, 1987). This renders the strain as a whole toxic to (non-immune) neighbours but imposes only a relatively small penalty on the growth rate per bacterium since only a few bacteria in the strain actually produce toxin and die. This situation is effectively modelled here; by assuming that the growth dynamics and mu-

\footnotetext{
*Colicinogenic bacteria are sometimes considered a model for altruistic behaviour: toxin producers commit suicide for the benefit of their conspecifics. Here, as in other models of altruistic behaviour, the spatial embedding results in the possibility of the evolution and maintenance of altruistic behaviour.
}

tation events affect the strains as a whole we simplify the model structure by interpreting the residents in single sites as single bacteria. Any group selective effects that single bacteria may experience as members of a strain are incorporated in the model through the effect of local growth which leads to patches of identical bacteria*.

The cellular automaton is a two-dimensional rectangular grid of $300 \times 300$ sites with periodic boundary conditions. The neighbourhood in which interactions between bacteria take place consists of the eight nearest neighbours of a site. A site is either empty or occupied by a bacterium. An empty site can be colonised by the "offspring" of a bacterium in a neighbouring site, resulting in two identical bacteria. The growth rate $R_{b}$ of (a strain of) wild-type bacteria, i.e. bacteria that do not carry any plasmids, is equal to one. If bacteria carry plasmids the growth rate is lowered by the number of toxin genes and immunity genes multiplied by the toxin penalty and the immunity penalty, respectively (see also Table 1). Actually, the effect of plasmids on the growth rate of their host may take many forms but in order to make the model as general as possible and to avoid any $a$ priori nonlinearities we used simply a linear relation.

The probability that a bacterium will grow in an empty site is equal to its growth rate divided by eight. We have used different rules to model the colonisation of empty sites, a cumulative colonisation rule and a probabilistic colonisation 
rule, but found no differences in the qualitative results of the model. The results reported here are based on the cumulative colonisation rule (probability of colonisation is equal to the sum of the growth rates of all eight neighbours).

There is a $10 \%$ probability that bacteria will die regardless of the plasmids that they carry. If in the neighbourhood of a bacterium toxins are produced for which the bacterium does not carry immunity genes its probability of death is increased by $30 \%$ per toxin gene. The results of the model do not depend much on the actual parameter values. We also studied the model with, for instance bacterium death rates as low as $1 \%$ and as high as $30 \%$ and mutation rates that differed several orders of magnitude from the one used here. However, in all cases the qualitative result of the model was the same as we report here. Table 1 gives the default parameter values, or range of parameter values that we used in the simulations reported here.

\section{Results}

We have studied the model for a large variety of parameter values. We found that the behaviour of the model can best be characterised in terms of two dynamical modes which we call hyperimmunity mode and multitoxicity mode. The two modes are determined primarily by the value of the growth penalty parameter $\left(P_{i}\right)$. In Section 3.2 we will show that the transition from one mode to the other with $P_{i}$ is very abrupt. First, however, we will describe two typical simulations of the model.

\subsection{TYPICAL TEMPORAL AND SPATIAL DYNAMICS}

Figure 1 shows time plots of simulations with growth penalties: (a) $P_{i}=0.06$ and (b) $P_{i}=0.10$
( $P_{t}=0.02$ for both simulations). The results are representative for the two dynamical modes.

The two upper panels in each figure show the bacterium population size. In panel 4 the population is partitioned into bacteria that carry $0,1, \ldots, 9$ number of immunity genes, in panel 3 the population is partitioned into bacteria that carry $0,1, \ldots, 9$ number of toxin genes. The two lower panels show cumulatively the filled plots of the number of toxin genes (panel 1) and the number of immunity genes (panel 2) of all plasmid types. Simulations are started with three out of nine plasmid types present in the population. The plasmids have either only an immunity gene or both immunity and toxin genes.

After the transients [in Fig. 1(a) after $t \approx 60000$, in Fig. 1(b) after $t \approx 5000$ ] several distinctive features are visible. In Fig. 1(a) $\left(P_{i}=0.06\right)$ a bacterium typically carries between six and nine immunity genes and between 0 and three toxin genes. Thus, bacteria carry many more immunity genes than toxin genes. In the lower panels we can see that at the population level the amount of toxin is very low and the immunity level is relatively high (note the difference in scale). This situation is very stable; all immunity gene types are always present, toxin gene types may temporarily disappear from the population but this happens very rarely.

In Fig. 1(b) $\left(P_{i}=0.10\right)$ the situation is quite different; a bacterium carries on average between four and six immunity genes and between three and six toxin genes. Here, bacteria carry just about as many immunity genes as toxin genes. In the lower two panels we see that compared with Fig. 1(a) the total level of toxin genes is much higher and the total level of immunity genes is much lower in the population. The temporal dynamics of plasmid types is much less stable;

FIG. 1. Temporal dynamics in (a) hyperimmunity mode $\left(P_{i}=0.06, P_{t}=0.02\right)$ and (b) multitoxicity mode $\left(P_{i}=0.10\right.$, $\left.P_{t}=0.02\right)$. The two lower panels show cumulatively the filled plots of the number of toxin genes (panel 1$)$ and the number of immunity genes (panel 2) per plasmid type (1-9). The two top panels show the bacterium population size, partitioned into bacteria that carry the specified number of toxin genes (panel 3) and immunity genes (panel 4), i.e. the distribution of toxin load and immunity load per bacterium (0-9). In panels 1 and 2 the colours denote different plasmid types, in panels 3 and 4 the colours denote the number of (toxin/immunity) genes carried per bacterium. In the hyperimmunity mode the transient is relatively long compared with the transient in the multitoxicity mode.

FIG. 5. Space-time plot showing invasions of colicins. Different colours denote bacteria that carry different combinations of plasmid genes: (a) invasion of plasmid results in complete turn over of the population [parameters as in Fig. 1(a)]; (b) invasion remains localized [parameters as in Fig. 1(b)]; (c) same state as in (b) except all bacteria are white; boundaries between different patches are often large gaps as a result of mutual killing. 


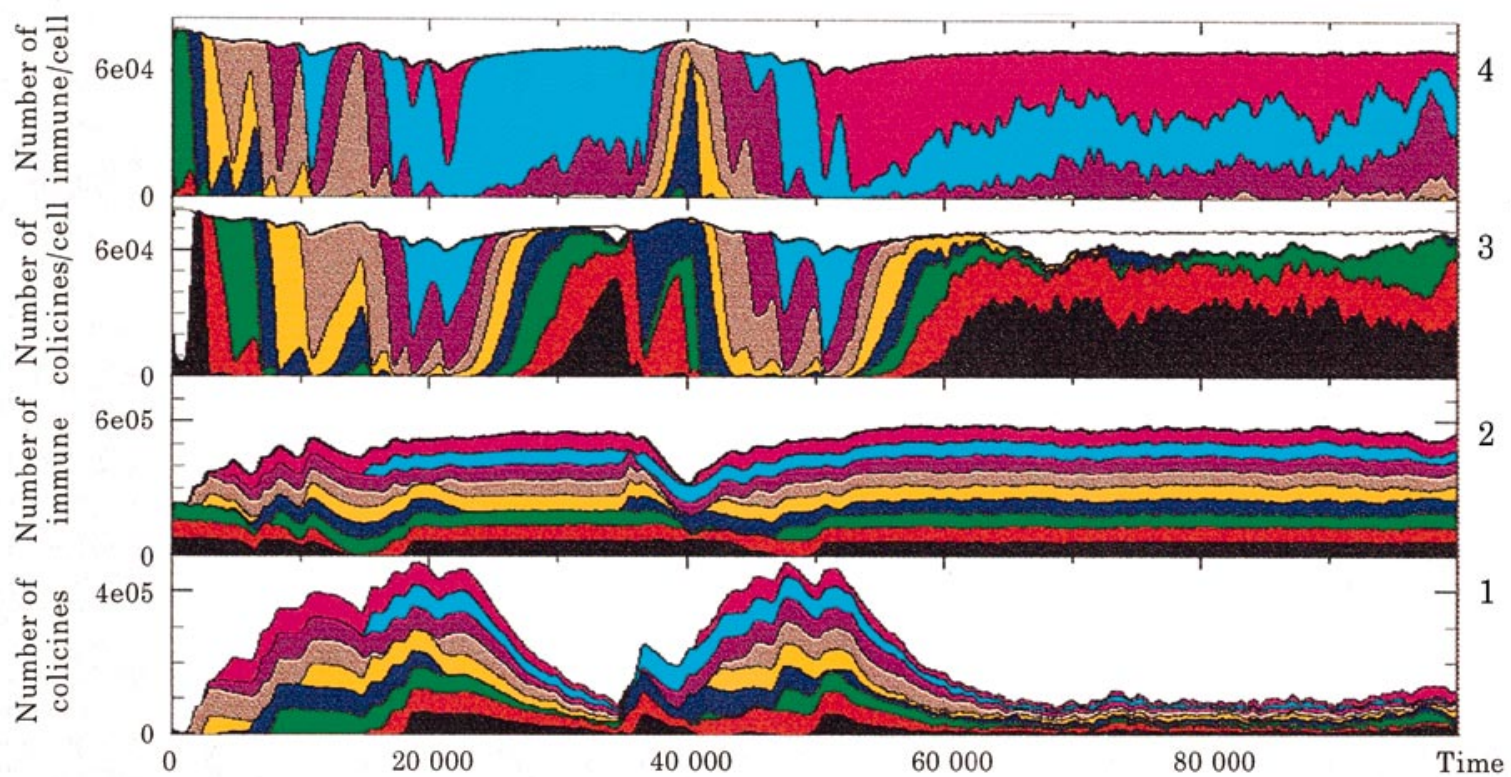

(1b)

Transient to multitoxicity mode

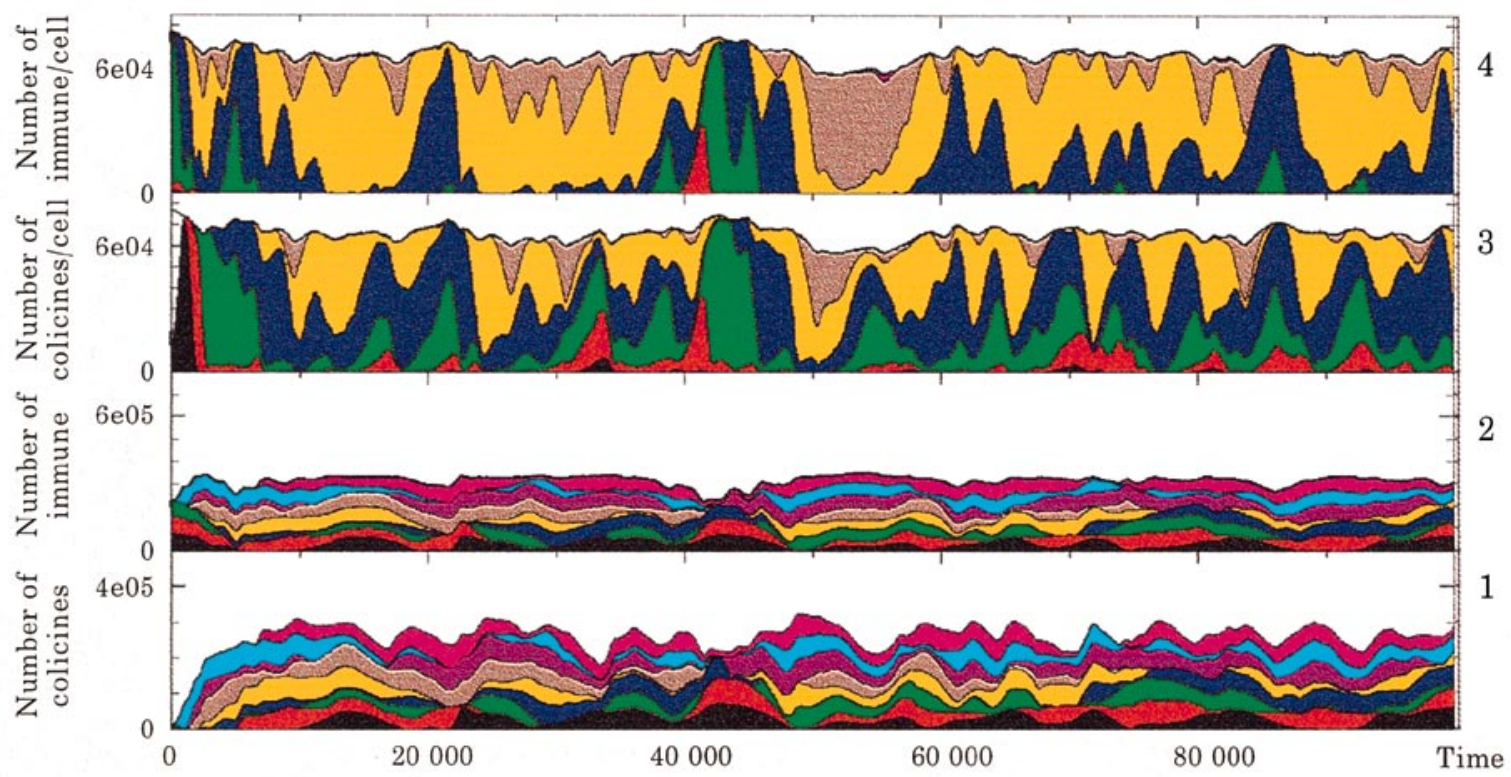

(Caption opposite)

Spacetime plots of hyperimmunity amd multitoxicity

(5a)

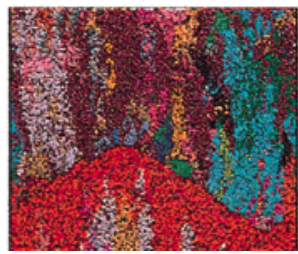

(5b)

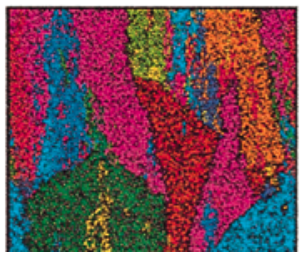

$(5 \mathrm{c})$

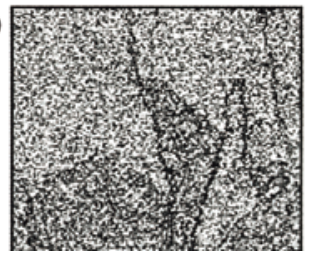

FIG. 5-(Caption opposite). 
toxin genes, and even immunity genes, frequently disappear from the population.

Thus, in the first case [Fig. 1(a)] all bacteria carry (almost) all immunity types but carry only a few or even no toxin genes. In the second case bacteria carry far fewer immunity genes but many more toxin genes than in the first case. The two dynamical modes also differ in how they are affected by invasions of new plasmids, in the transient from the initial situation, and in the temporal and spatial dynamics of different bacterium types. We return to these issues in Section 3.3. First we show that the dynamical modes as shown here reflect instances of two different phases in the model behaviour which are separated by a sharp transition.

\subsection{HYPERIMMUNITY AND MULTITOXICITY: A PHASE TRANSITION}

In Fig. 2 we plot the size of the bacterium population and the mean number of toxin and immunity genes per bacterium for a range of growth penalties $P_{i}$. The figure shows three panels for different values of $P_{t}$. For all values of $P_{t}$ a phase transition occurs for $P_{i} \approx 0.07$. The dynamics in the model to the left and right of the phase transition is characterised by:

- the bacterium population size; on the left it decreases slightly with $P_{i}$ while on the right it is independent of $P_{i}$;

- the mean number of immunity genes per bacterium; this number is independent of $P_{i}$ on the left but decreases with $P_{i}$ on the right;

- the mean number of toxin genes per bacterium; this number increases somewhat with $P_{i}$ on the left but decreases with $P_{i}$ on the right;

- the difference between immunity level and toxin level per bacterium; bacteria on the left have a much higher level of immunity than level of toxin; on the right the two are about the same;

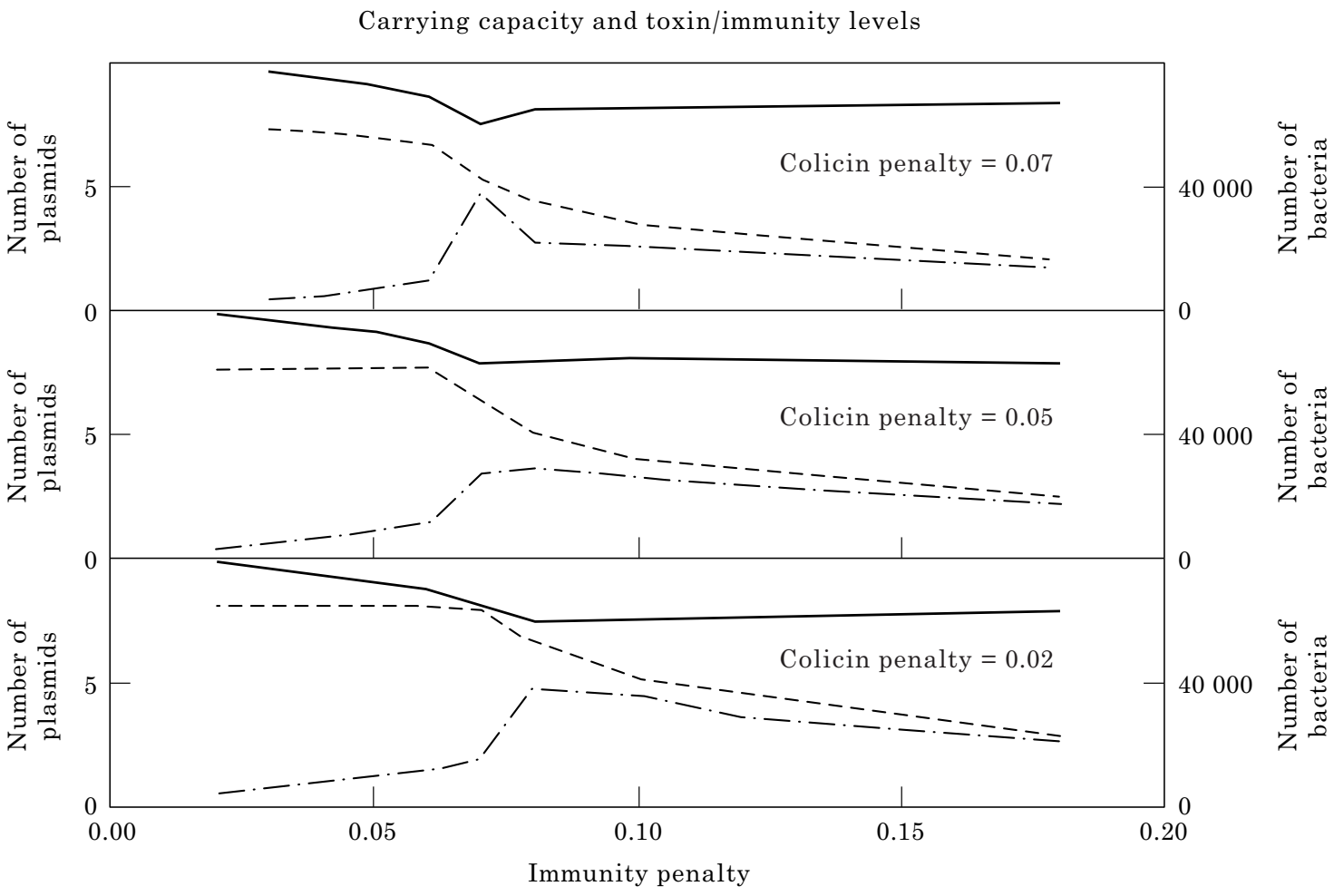

Fig. 2. Population size, immunity levels and toxin levels for a range of immunity growth penalties $P_{i}$. From top panel to bottom panel: $P_{t}=0.07 . P_{t}=0.05, P_{t}=0.02$. For low $P_{i}$ the bacteria are hyperimmune; bacteria have high immunity levels and low toxicity levels. For high $P_{i}$ the bacteria are multitoxic; bacteria have lower immunity levels but higher toxicity levels. (- --$)$ Number of immunity genes per bacterium; $(-\cdot-\cdot-)$ number of toxin genes per bacterium; $(-)$ number of bacteria. 


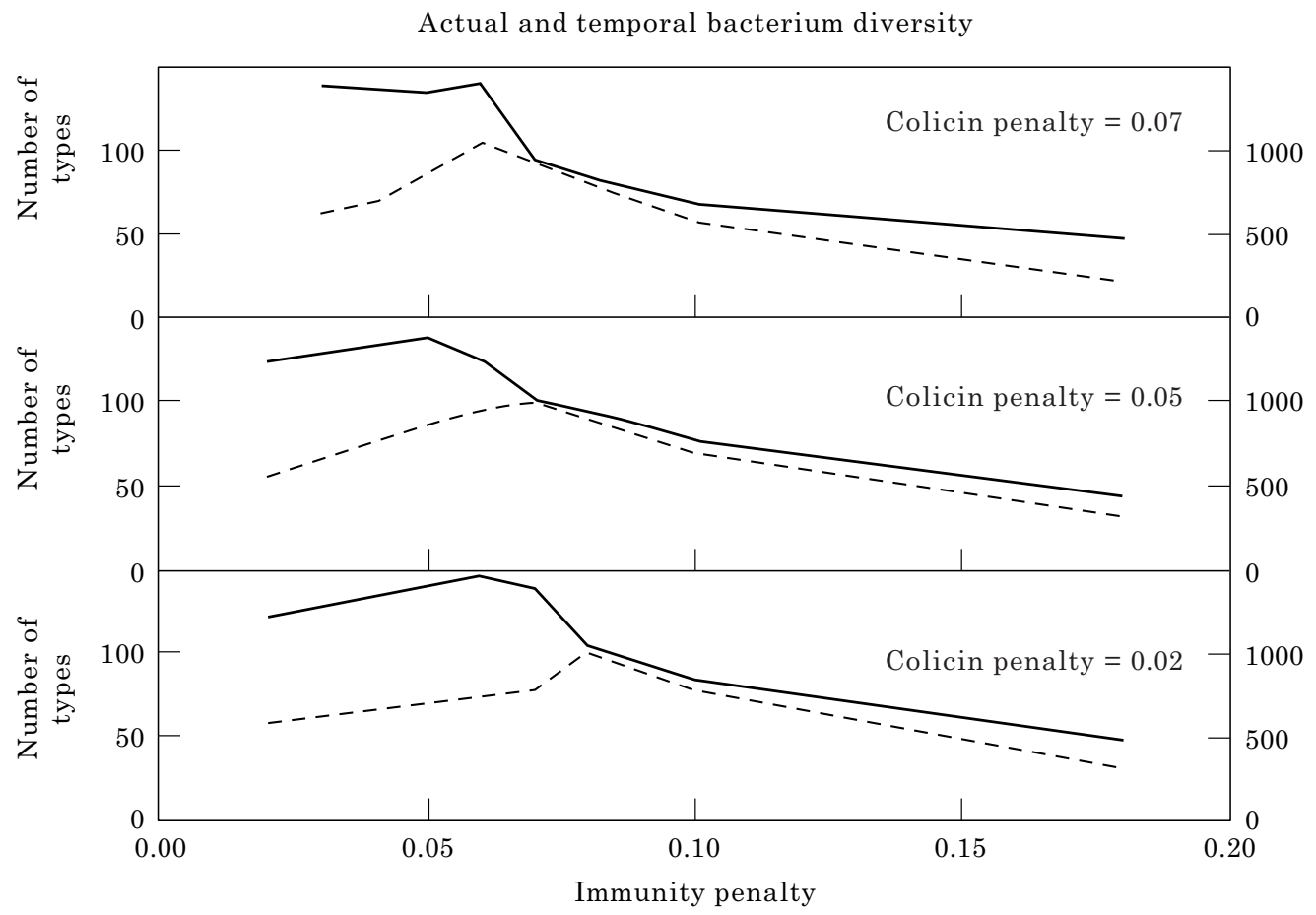

FIG. 3. Bacterium diversity for a range of immunity growth penalties. Shown are the average number of bacterium types per generation and the total number of bacterium types over 10 samples. From top panel to bottom panel: $P_{t}=0.07$, $P_{t}=0.05, P_{t}=0.02$. For all $P_{i}$ average and total diversity is high. For low $P_{i}$ the difference between the two lines indicates that a large proportion of the bacteria are residents. For high values of $P_{i}$ all bacteria are transients. ( -$)$ Number of types in population; (-- ) number of types in 10 samples.

at the transition the toxin level rises abruptly.

Thus, for low $P_{i}$ an increase in the growth penalty of the plasmids results in a decrease in carrying capacity of the bacteria while the number of toxin genes per bacterium rises and the number of immunity genes per bacterium remains equal. Here, it is the bacteria that "pay" the price of an increasing cost of the plasmids. For high $P_{i}$, however, the increase in penalty leads to a decrease in immunity while the carrying capacity of the bacterium population remains equal. In this case it is the plasmids that pay the price.

In Fig. 3 we plot the diversity of bacterium types. A bacterium type is defined by the ensemble of plasmid genes that it carries. Given that a plasmid can be in three states (absent, toxic-immune, or immune-only) the upper bound for total number of possible bacterium types is $3^{9}=19683$. However, this upper bound includes bacteria with a very small number of immunity genes and bacteria with a very large number of toxin genes, neither of which occur in the model in either of the two modes. We plot the average number of bacterium types per time-step (solid line) and the total number of bacterium types found in 10 samples taken 10000 time-steps apart (dashed line). Both lines indicate that a high diversity of the bacterium population is easily maintained at any one time and over longer time periods. Moreover, we see a phase transition occurring for $P_{i} \approx 0.07$ again. Interestingly, the difference between the average number of types and the actual number of types changes from a factor two to almost zero. Thus, for lower values of $P_{i}$ a large proportion of the bacteria in the population are residents. For larger values of $P_{i}$ on the other hand almost all bacterium types are transient; in each sample the population is completely replaced by new types of bacteria.

\subsubsection{Carrying capacity, growth-rate and the phase transition}

The carrying capacity of a population that grows in space depends nonlinearly on the growth-rate per individual. The phase transition 
in the behaviour of the model can be understood if this dependence is taken into account.

If we measure the carrying capacity of a population in a simple spatially explicit growth model we get the relation as it is plotted in Fig. 4.* For high growth rates the carrying capacity is almost independent of the growthrate. In a relatively small parameter region, however, the carrying capacity of the population collapses rapidly with decreasing growth-rate.

The phase transition in the colicin model corresponds to the collapse in the carrying capacity in Fig. 4. The $x$-axis in this figure is scaled such that it corresponds to the growth rate of a bacterium that carries eight immunity genes and one toxin gene $\left(P_{t}=0.02\right.$, see the growth rate equation in Table 1). The dependence of the carrying capacity on the growth rate leads to the following observation. If the growth rate has little effect on the carrying capacity, i.e. $P_{i}<0.07$, bacteria maintain high numbers of immunity genes per bacterium, independent of the growth penalty per gene. For $P_{i}>0.07$ this option is no longer viable. However, instead of gradually lowering the number of immunity genes in order to maintain a sufficient growth rate the strategy of bacteria in coping with the plasmids changes dramatically. For high values of $P_{i}$ the bacteria carry simply as many complete plasmids (i.e. both toxin and immunity genes) as possible. In this regime the number of plasmids carried per bacterium drops with $P_{i}$.

*In fact, in a mean field approximation (i.e. $\mathrm{d} X / \mathrm{d} t=b X(S-X)-\mathrm{d} X$, with $b=$ birth rate, $d=$ death rate, $S=$ size of space) the carrying capacity shows the same, nonlinear relation to the growth rate; $\tilde{X}=1-d / b S$.
The dynamics for low values of $P_{i}$ and for high values of $P_{i}$ are very typical. We will refer to these two modes as the hyperimmunity and the multitoxicity mode. Of course many variations on the parameter settings that we used for these simulations are possible, but we found that in all simulations that we performed the dynamics could best be characterized with respect to these two modes. In short, hyperimmunity is characterized by high immunity levels, low toxicity levels and relatively stable dynamics. Multitoxicity is characterized by levels of immunity and toxicity which are about equal per bacterium and the dynamics are less stable; both toxin genes and immunity genes may disappear temporarily from the population.

\subsection{PLASMID INVASIONS}

The transients in the two simulations in Figs 2 and 3 help to characterize the two modes further. If a certain plasmid type is completely absent from the population, or if a plasmid is of a novel type, invasions of bacteria that carry the plasmid can have drastic effects on the dynamics since none of the bacteria present in the population is immune to the invading plasmid. However, the hyperimmunity and the multitoxicity modes react differently to such invasions.

In Fig. 1(a) up to $t \approx 60000$ invasions of new plasmid types lead to large disruptions in the population. An invasion at $t \approx 35000$ is particularly representative. At $t \approx 20000$ the type 4 (i.e. blue) plasmid disappears from the population. After that the dynamics tend to settle down in the characteristic behaviour; all remaining immunity and toxin gene types are present, the immunity level per bacterium is high

Carrying capacity

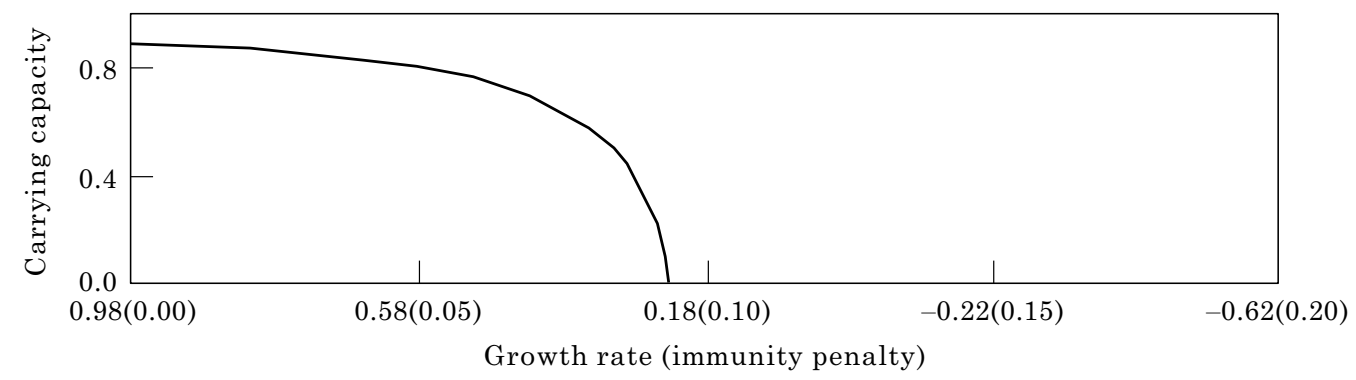

FIG. 4. Carrying capacity of a simple locally growing population vs. growth rate. The $x$-axis corresponds to the $x$-axis of Figs 2 and 3 for bacteria that carry eight immunity genes (penalty values between brackets) and one toxin gene $\left(P_{t}=0.02\right)$. 
and toxin level per bacterium goes down. But at $t \approx 35000$ plasmid type 4 comes back by generative mutation. The bacterium strain that carries this plasmid takes over the whole population. The result of the invasion is that all other toxin types, except type 1 (i.e. black) and 2 (i.e. red), are expelled from the population because the invading strain does not carry these toxin genes but it does carry the immunity genes. The immunity gene types of the expelled toxin types now no longer come into contact with the corresponding toxins, so they too are slowly expelled from the population. More invasions follow until at $t \approx 60000$ the dynamics are stabilized.

In Fig. 1(b) we see that the transient is much shorter. Contrary to the previous case, in the multitoxicity mode bacteria do not carry all immunity genes. In general, the bacteria carry only those immunity gene types for which they also carry the toxin genes. Thus, patches of bacteria that carry different ensembles of plasmids are often sensitive to some of each other's toxins. At the boundary between patches this leads to mutual killing of bacteria of the different patches.

In Fig. 5 we show spacetime plots of invasions by plasmids of the bacterium population in the hyperimmunity mode (a) and multitoxicity mode (b). In the spacetime plot we draw horizontal cross-sections of the grid for every 10th time-step. The different colours represent different bacterium types (see Section 3.2).

In Fig. 5(a) a red patch appears; it denotes a bacterium strain that carries a plasmid that is new in the population. The patch takes over the population completely on a short time-scale. The subsequent competition between this bacterium type and derived bacterium types that lack toxin genes (but not immunity genes) is on a much longer time-scale.

In Fig. 5(b) a red patch also appears, again denoting a bacterium strain that carries a new plasmid*. In this case the invading bacterium strain does not take over the population. In fact, in one direction it grows at roughly the same

\footnotetext{
*The red, invading bacteria in Fig. 5(a) and (b) are of different types. Both are drawn with the same colour for clarity.
}

speed as the invading patch in the hyperimmunity mode, but in the other direction it is itself taken over by a bacterium strain that was already present in the population.

Figure 5(c) is the same spacetime plot as Fig. 5(b) except that all bacteria are coloured white. Many boundaries between patches in Fig. 5(b) are actually large gaps that have resulted from the mutual killing. Since an invading bacterium strain does not carry all immunity genes it will at some point encounter bacteria that produce toxins against which it is not immune. As a result invasions will not spread out over the whole population. Although in the hyperimmunity mode the immunity level per bacterium is very high the multitoxicity mode seems more robust to invasions.

Once the hyperimmunity mode is stabilised it is independent of the creation of plasmids. In the multitoxicity mode plasmid types often disappear from the population. Only by continuous generation of new plasmids are the dynamics maintained in the long run. If absent plasmids are not reintroduced the bacteria keep losing plasmids up to the point where they can attain hyperimmunity mode behaviour with the available plasmid types. That is because fewer plasmid types "exist" bacteria can carry all immunity genes that are present while maintaining a viable growth rate. The rate at which plasmids are generated, i.e. the generative mutation rate $\mu_{g}$, and the rate at which plasmid genes are mutated, i.e. the degenerative mutation rate $\mu_{d}$, only weakly influence the results we have reported. In fact, the mutation rates scale with the size of the spatial grid. A minimum grid size, or $\mu_{d}$, is necessary for all plasmid types to fit in stably. If either is too small the hyperimmunity mode will not remain stable because of continued invasions and expulsion of plasmids in the population, as mentioned before.

\section{Discussion}

\subsection{PLASMID DIVERSITY}

In our model we see that at the level of the bacterium population a high diversity of plasmids is easily maintained under a wide variety of parameter settings. An important 
feature of our model in this respect is the spatial embedding of the population of bacteria. In fact, if we perform global mixing in the model the population becomes completely homogeneous due to global competitive exclusion.

If we look at the competition between complete $^{*}$ plasmids in the spatial model we see that plasmids cooperate rather than compete. In the parameter region in which we might expect to find the hyperimmunity mode we find that a decrease in the growth rate of the bacteria leads to an increase in the number of plasmids. For this phenomenon it makes no difference whether the decrease in the growth rate of the bacteria is imposed directly or as a result of increasing the number of plasmids the bacteria carry or increasing the growth penalty per immunity gene (e.g. see Fig. 2). The bacteria decrease their carrying capacity with increasing cost per immunity gene rather than reduce the number of immunity genes. Thus, the bacteria can be said to integrate information with respect to colicin types over many generations. Although individual bacteria encounter only a small number of colicin types in their lifetime they nevertheless maintain a high immunity level as a result of spatial and temporal information integration (see Pagie \& Hogeweg, 1997).

In the parameter region in which bacteria cannot carry the immunity genes of all types, i.e. the multitoxicity mode, plasmids are better off if they are in the presence of other plasmids as well. If we remove plasmid types in this parameter region the state of the bacterium population changes from the multitoxicity mode to the hyperimmunity mode, where there are high numbers of immunity genes but low numbers of toxin genes, or complete plasmids. Thus, from the point of view of the complete plasmids it is always advantageous to be with many other types of plasmids rather than only a few.

In the hyperimmunity mode plasmids only interact with each other via the growth rate of the host bacterium. In the multitoxicity mode, on the other hand, plasmids cooperate in a more direct manner. Here, plasmids are members of an

\footnotetext{
*Complete plasmids consist of both the toxin gene and the immunity gene. Immunity-only plasmids are in themselves non-viable in the long run.
}

ensemble of plasmids that are carried in one bacterium which therefore produces a combination of toxins. In Section 3.3 we showed that competition between different ensembles of plasmids can lead to mutual killing of bacteria. As a result plasmids, by being members of an ensemble rather than being alone, have additional protection against other competing plasmid ensembles. Of course competition, here, at the level of plasmid ensembles is harsh. In the hyperimmunity mode plasmid ensembles are small and hardly ever come into contact.

\subsection{THE COST OF DEFENCE}

We found that plasmids in general fare well if their corresponding immunity genes are readily "lost" by the bacterium host. Clearly, complete plasmids will always be in the neighbourhood of bacteria that carry immunity-only plasmids since these are one of the viable mutants of the complete plasmids. Thus, bacteria that carry complete plasmids will always find themselves in the neighbourhood of bacteria that are insensitive to their toxin and are better competitors. Unless toxin-producing bacteria can invade patches of sensitive bacteria the toxin-producing bacteria will be outcompeted. Toxin-producing bacteria invade patches of sensitive bacteria very fast. The time-scale of the competition between the remaining combinations of bacterium variants (e.g. sensitive vs. immunity-only bacteria) is much longer because it is based only on differences in growth rate. The speed at which the immunity-only bacteria are overtaken by the sensitive bacteria is the primary limiting factor for the number of toxin-producing bacteria. Thus, a plasmid increases its chance of long-term survival by making it very advantageous for immunity-only bacteria to lose their immunity gene also. The plasmids can do this by imposing on the host a high growth penalty as a result from carrying the immunity gene. Under what circumstances a high immunity penalty can evolve remains an open question since both bacteria and plasmids experience a short-term benefit of low costs. From other systems we know, however, that as a result of spatial dynamics long-term benefits may prevail over short-term benefits (Savill et al., 1997; Boerlijst \& Hogeweg, 1991). 


\subsection{EXPERIMENTAL DATA}

The previous sections have suggested that it is advantageous for the plasmids to impose high growth penalties on the host. From the point of view of the bacterium it seems advantageous to have a large growth rate. However, the reduction in the carrying capacity due to a decrease in growth-rate is small (see Figs 2 and 4).

Experimental data suggest that in natural isolates the proportion of bacterium strains that produce one or more colicins is high [51\% Achtman et al. (1983) and $35 \%$ in Riley \& Gordon (1992)], and resistance to colicins is also high $[22 \%$ of the bacteria in Riley \& Gordon (1992) are resistant to all colicin types tested]. In our model we find that in the hyperimmunity mode the percentage of bacteria that produce one or more colicins varies between $38 \%\left(P_{i}=0.02\right.$, $\left.P_{t}=0.02\right)$ and $99 \%\left(P_{i}=0.07, P_{t}=0.02\right)$. The immunity levels in the hyperimmunity mode are high; bacteria are on average immune to $90 \%$ of the plasmid types and $32 \%$ of the bacteria are immune to all plasmid types $\left(P_{i}=0.02\right.$, $\left.P_{t}=0.02\right)$. In the multitoxicity mode, on the other hand, we find that each bacterium produces several colicins. Also, the immunity level per bacterium is much lower than in the hyperimmunity mode (e.g. see Fig. 2). Finally, in the multitoxicity mode the bacteria are generally not immune to any more colicin types than they actually produce.

These data suggest that in natural circumstances the growth penalty imposed by plasmids falls well within the hyperimmunity mode. In fact, it is shown that a significant number of bacteria do not carry any plasmids $[14 \%$ in Riley \& Gordon (1992), 8\% in Caugant et al. (1981) and $4 \%$ in Achtman et al. (1983)]. Assuming that the immunity against colicins is coded on plasmids, the absence of plasmid makes sense in the (local) absence of colicin producing plasmids only. The results of our model differ most from the experimental data with respect to the number of wild-type bacteria, i.e. bacteria that do not carry any plasmid at all. One possible mechanism that may influence this discrepancy between our results and the experimental data is the active removal by bacteria of all plasmids at one time through "spitting". This would "convert" the bacteria directly into a wild-type state. However, if we include in our model the possibility of "spitting" we see that the numbers of wild-type bacteria are in fact very low $\left(<3 \% ; P_{i}=0.02\right.$, $P_{t}=0.02$, spit-rate $\left.=0.01\right)$. The number of colicin-producing bacteria, however, increases from $38 \%$ without spitting to $73 \%$ for high spit-rates. In the multitoxicity mode, on the other hand, the inclusion of spitting in the model does not have any effect other than a slight increase in the death rate of bacteria due to unwarranted loss of immunity.

Other than the difference in number of wild-type bacteria mentioned above the agreement between the experimental data and our results is striking, although experimental data are still somewhat scarce. Most experimental studies report only on colicin production and not on potential additional immunity. Moreover, in general they do not differentiate between immunity and other means of defence. Finally, it is not clear whether natural bacterium populations in different nutritional conditions adopt different strategies with respect to colicin plasmids; our results suggest that this might be the case.

\section{Conclusion}

We have studied the interaction between multiple colicin plasmid types and bacteria in a spatially explicit model. We have shown that a high diversity of colicin plasmids is easily maintained under many parameter settings. The most important finding in our study is the occurence of a phase transition in the colicinogenic dynamics. This transition is dependent on the growth penalty caused by immunity production. We have shown that if the cost of immunity is such that a bacterium can carry most types of immunity without a large reduction of its carrying capacity, it will actually do this. Simultaneously this will minimise the number of toxin genes per bacterium. We call this hyperimmunity. When the cost of carrying all immunity gene types becomes too high for the bacteria to remain viable they will then switch to the multitoxicity behaviour. In this mode bacteria carry as many complete plasmids as possible. At the phase transition the immunity 
level per bacterium drops whereas the toxin level per bacterium rises (Fig. 2).

We have shown that in our model it is advantageous for plasmids to impose a high growth penalty on their host that results from the defence mechanism that defends the host to the rather unpleasant action of the plasmid. The high cost of the defence mechanism ensures that a plasmid-variant that only defends its host against toxins without coding for the toxin will quickly be outcompeted by wild-type bacteria. The presence of wild-type bacteria gives the plasmid an opportunity to remain in the population. Other systems that also comprise a threat plus defence should also benefit from the high cost of the defence mechanism.

The authors thank S. M. McNab for linguistic advice. They also thank the referees for constructive criticism and helpful suggestions. The investigations were supported by the Life Sciences Foundation (SLW), which is subsidized by the Netherlands Organization for Scientific Research (NWO).

\section{REFERENCES}

Achtman, M., Mercer, A., Kusecek, B., Pohl, A., Heuzenroeder, M., Aaronson, W., Sutton, A. \& Silver, R. P. (1983). Six widespread bacterial clones among Escherichia coli $\mathrm{K} 1$ isolates. Infect. Immun. 39, 315-335.

Boerlijst, M. C. \& Hogeweg, P. (1991). Self-structuring and selection: spiral waves as a substrate for evolution. In: Artificial Life (Langton, C. G., ed.) Vol. 2, pp. 255-276. Redwood City, CA: Addison-Wesley.

Bouma, J. E. \& Lenski, R. E. (1988). Evolution of a bacteria/plasmid association. Nature 335, 351-352.

Caugant, D. A., Levin, B. R. \& Selander, R. K. (1981). Genetic diversity and temporal variation in the $E$. coli population of a human host. Genetics 98, 467-490.

Chao, L. \& Levin, B. R. (1981). Structured habitats and the evolution of anti-competitor toxins in bacteria. Proc. Nat. Acad. Sci. U.S.A. 78, 6324-6328.

CoOper, P. C. \& James, R. (1984). Two new E colicins, E8 and E9, produced by a strain of Escherichia coli. J. Gen. Microbiol. 130, 209-215.

Durrett, R. \& LEVIN, S. (1997). Allelopathy in spatially distributed populations. J. theor. Biol. 185, 165-171.
Dykes, G. A. \& Hastings, J. W. (1997). Selection and fitness in bacteriocin-producing bacteria. Proc. R. Soc. Lond. B. Biol. Sci. 264, 683-687.

Feldgarden, M., Golden, S., Wilson, H. \& Riley, M. A. (1995). Can phage defence maintain colicin plasmids in Escherichia coli? Microbiology 141, 2977-2984.

FrANK, S. A. (1994). Spatial polymorphism of bacteriocins and other allelopathic traits. Evol. Ecol. 8, 369-386.

HARDY, K. G. (1975). Colicinogeny and related phenomena. Bacteriol. Rev. 39, 464-515.

Hartl, D. L. \& Dykhuizen, D. E. (1984). The population genetics of Escherichia coli. Annu. Rev. Genet. 18, 31-68.

Iwasa, Y., Nakamaru, M. \& Levin, S. A. (1998). Allelopathy of bacteria in a lattice population: competition between colicin-sensitive and colicin-producing strains. Evol. Ecol. (in press).

James, R., Kleanthous, C. \& Moore, G. R. (1996). The biology of $\mathrm{E}$ colicins: paradigms and paradoxes. Microbiology 142, 1569-1580.

KonISKY, J. (1982). Colicins and other bacteriocins with established modes of action. Annu. Rev. Microbiol. 36, 125-144.

Lau, P. C., Rowsome, R. W., Watson, R. J. \& Visentin, L. P. (1984). The immunity genes of colicins E2 and E8 are closely related. Biosci. Rep. 4, 565-572.

LeVIN, B. R. (1988). Frequency-dependent selection in bacterial populations. Phil. Trans. R. Soc. Lond. B. Biol. Sci. 319, 459-472.

Luria, S. K. \& SuIT, J. L. (1987). Colicins and col plasmids. In: Escherichia Coli and Salmonella Typhimurium: Cellular and Molecular Biology (Neidhardt, F. C., ed.) Vol. 2, Chap. 102. pp. 1615-1624. Washington, D.C.: American Society for Microbiology.

Pagie, L. \& Hogeweg, P. (1997). Evolutionary consequences of coevolving targets. Evol. Comput. 5(4), 401-418.

Pugsley, A. P. (1984). The ins and outs of colicins. Part II. Lethal action, immunity and ecological implications. Microbiol. Sci. 1, 203-205.

Riley, M. A. (1993). Molecular mechanisms of colicin evolution. Mol. Biol. Evol. 10, 1380-1395.

Riley, M. A. \& Gordon, D. M. (1992). A survey of Col plasmids in natural isolates of Escherichia coli and an investigation into the stability of Col-plasmid lineages. $J$. Gen. Microbiol. 138, 1345-1352.

Savill, N. J., Rohani, P. \& Hogeweg, P. (1997). Self-reinforcing spatial patterns enslave evolution in a host-parasitoid system [published erratum appears in J. theor. Biol. 1997 188(4), 525-526]. J. theor. Biol. 188, 11-20.

Singh, M., Ahmad, C. M. \& Yadava, J. N. (1989). Drug resistance and colicin production among various serotypes of Escherichia coli of animal origin. Ind. J. Pathol. Microbiol. 32, 161-166. 